\title{
The Impacts of a Systematic Exercise Method on Physical Activity and Psychological Factors
}

\author{
Jung-Ho Lee \\ Professor, 219-705 Dept. Physical Therapy, Kyungdong Univ., Bongpo-ri, \\ Toseong-myeon, Goseong-gun, Gangwon-do, Republic of Korea \\ ljhcivapt@naver.com
}

\begin{abstract}
This study was conducted to investigate the impacts of a systematic exercise method on physical activity and psychological factors. This study applied a systematic exercise method for 4 weeks. In this study, to apply a systematic exercise method, various exercise methods applying resistance and a method applying unstable base surface were used. The evaluation was conducted by dividing physical activity and psychological state into pre-test and post-test. In the study results, there was a statistically significant difference between the pre-and postevaluation. In conclusion, the application of a systematic exercise method has a positive effect on physical activity and psychological factors.
\end{abstract}

Keywords: Systematic exercise, Physical activity, Resistance

\section{Introduction}

Skeletal muscles account for about $40-50 \%$ of a person's total body mass and decrease by $3-$ $10 \%$ every 10 years after age 25, during which mitochondrial functions also decrease. Weakening of skeletal muscles causes muscular system disorders and is a prominent symptom among people with neurological disorders [1]. The change in muscle strength due to the weakening of muscles is used as a sensitive indicator for the aging process. Muscle weakness in elderly people is directly related to the movement of the upper limbs and the mobility of the lower limbs for them to live an independent life; thus, maintaining a state without muscle defects is important for preventing and delaying the physical weakening process [2].

To maintain posture and perform voluntary movements in daily life, posture stability and balance ability are required for the body to respond properly to external changes. Several studies on the elderly have reported that the most common causes of injuries in elderly people are their decreased ability to control balance and impaired functional motility. Fall is a common problem in the elderly and is one of the major causes of increased morbidity and mortality [3].

Falling accidents can occur throughout all life cycles, with a higher incidence, especially in the elderly over the age of 65 . Most elderly people experience a decline in balance ability, which causes a fall; the risk of falling is 10 times higher in the elderly than in other age groups, and $30 \%$ of the elderly over the age of 65 experience falling accidents at least once a year. While interacting among the motor systems, balance ability detects stimuli caused by the external environment, recognizes changes in body orientation, and maintains the center of gravity. The maintenance of balance is associated with stabilization and contraction control of the upper and lower limb muscles through the interactions between vision, cerebellar function,

Article history:

Received (April 4, 2021), Review Result (May 5, 2021), Accepted (July 10, 2021) 
vestibular sensations, and the proprioceptive sensory system. To maintain balance, normal motor skills, sensory skills, and cognitive skills of the brain, which play an upper-level central role, are required [4][5].

As the elderly population increases due to advances in medicine, many studies are being conducted on elderly people. Most previous studies focused on the improvement of muscle strength and balance after engaging in only fragmentary exercise programs; thus, studies examining the effects of comprehensive exercise programs are insufficient [6].

Therefore, this study designed a new group exercise program that included several individual exercise programs aimed at increasing muscle strength, balance, flexibility, and gait function. The effects of the program on the muscle strength, balance, gait, quality of life, and fall-related efficacy of elderly people, as well as potential gender-related effects, were investigated.

\section{Methods}

\subsection{Subjects}

This study was conducted on 30 elderly people who participated in the elderly health class at welfare centers through public recruitments and registrations at local public health centers. In the course of the study, six people dropped out due to non-participation in the program, difficulties in performing an exercise, and personal circumstances, leaving a total of 24 participants in the study.

Before starting, the purpose and method of the study were sufficiently explained to all subjects, and the study was conducted after they provided voluntary consent to participate [Figure 1]. All experimental procedures were performed by the Declaration of Helsinki.

\subsection{Systematic exercise methods}

The systematic exercise method was performed twice a week for 4 weeks. The method consisted of four stages, including the 1st-week program, the 2nd-week program, the 3rd-week program, and the 4th-week program, according to the level of difficulty.

The comprehensive exercise program consisted of exercise using the body as resistance (E1), exercise using a resistance band (E2), and exercise using a balance pad (E3). E1 consisted of six movements (heel lift, four-way stepping, squat, lunge, core, and bridge). E2 was performed to enhance the muscle strength of the upper and lower limbs and the abdomen. Exercises for the upper limbs consisted of movements around the shoulders, elbows, and wrists. In E3, a pad was placed on the base surface where the exercise using the body as resistance and the exercise using the resistance band were performed. 


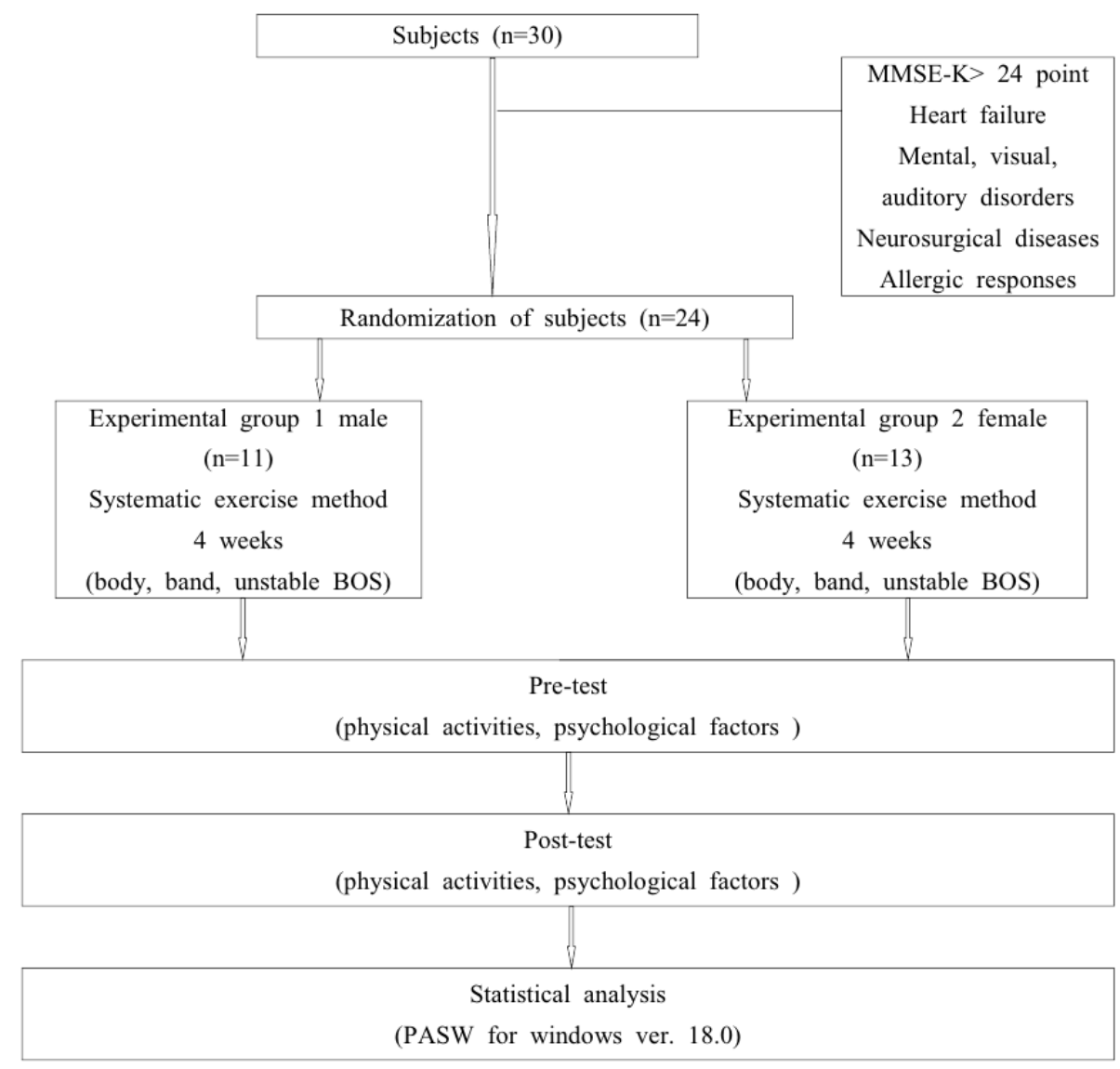

Figure 1. Flowchart depicting all of the Declaration of Helsinki's experimental protocols

\subsection{Evaluation methods}

\subsubsection{Muscle strength}

To measure the muscle strength of elderly subjects, their grip strength was measured. The grip strength was measured using a digital grip dynamometer (T.K.K.K 5401 Grip Strength Dynamometer, Takei Scientific Instruments, Japan).

\subsubsection{Balance}

Berg balance scale (BBS) is a tool that objectively measures static and dynamic balance. The BBS assessment tool consists of 14 functional tasks that are generally performed in daily life, in which a minimum of 0 to a maximum of 4 points are assigned to three areas-sitting, standing, and posture change - for a total of 56 points.

\subsubsection{Gait}

In the timed up and go (TUG) test, the subject sat on a chair with armrests, then with a command of 'start', got up from the chair, walked straight up to a mark 3 meters away, and returned to sit back on the chair. 


\subsubsection{Quality of life}

In this study, a health-related quality of life (SF-36) evaluation sheet was used to assess the quality of life. The SF-36 consists of 36 questions, each measuring eight health concepts, and includes one multiple question area.

\subsection{Analysis}

All statistical analyses in this study were performed using PASW 18.0 to calculate the mean values and standard deviations for each measurement item. To compare the differences before and after the exercise program, a paired sample t-test was conducted, and an independent t-test was conducted to compare the inter-group. All statistical significance levels of the data were set to $\leq 0.05$.

\section{Results}

Regarding gender, men showed statistically significant changes in grip strength and quality of life compared to women $(\mathrm{P}<0.05)$, but there were no significant differences in balance, gait, and fall-related efficacy between males and females $(\mathrm{P}>0.05)$ [Figure 2,3,4,5].

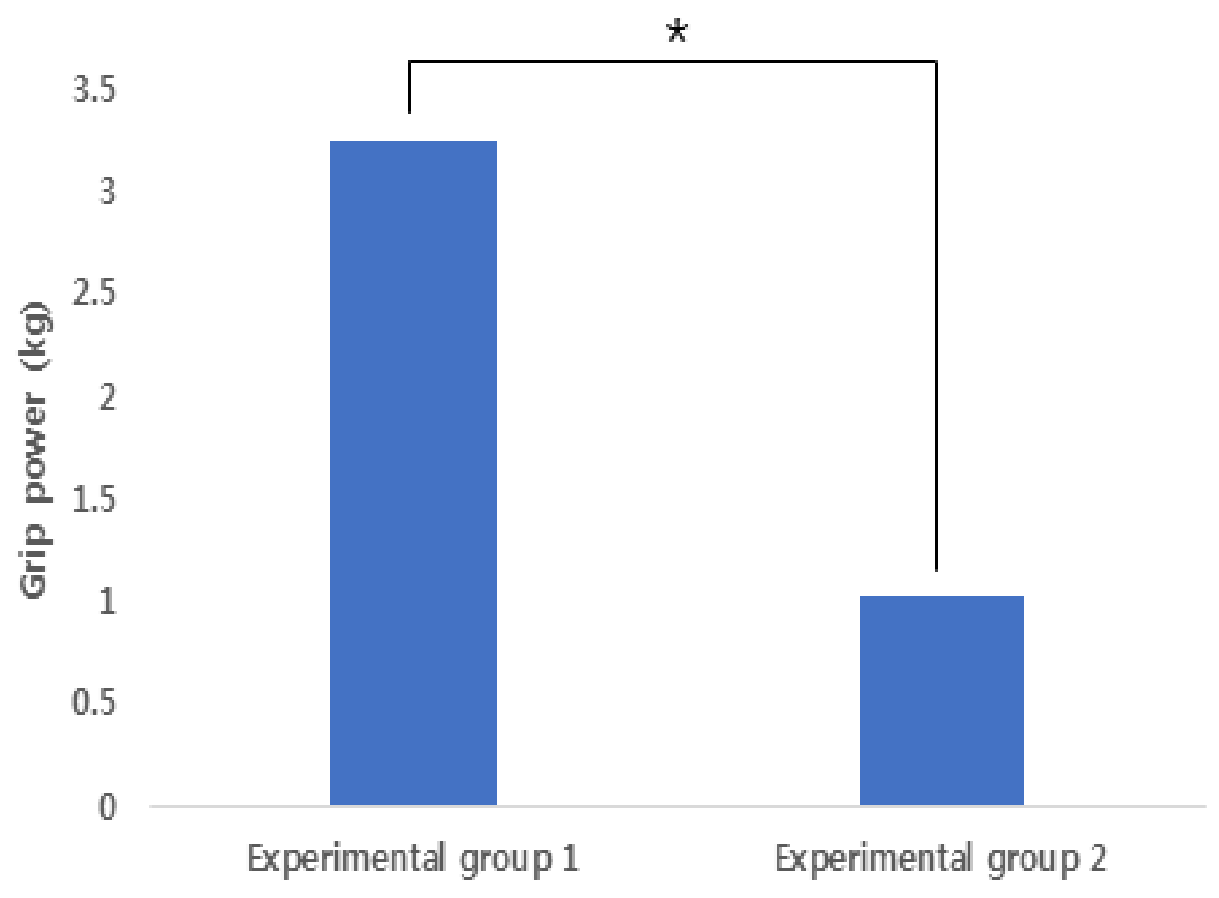

Figure 2. Muscle strength

${ }^{*} p<0.5$, Mean $\pm S D:$ Mean \pm standard deviation 


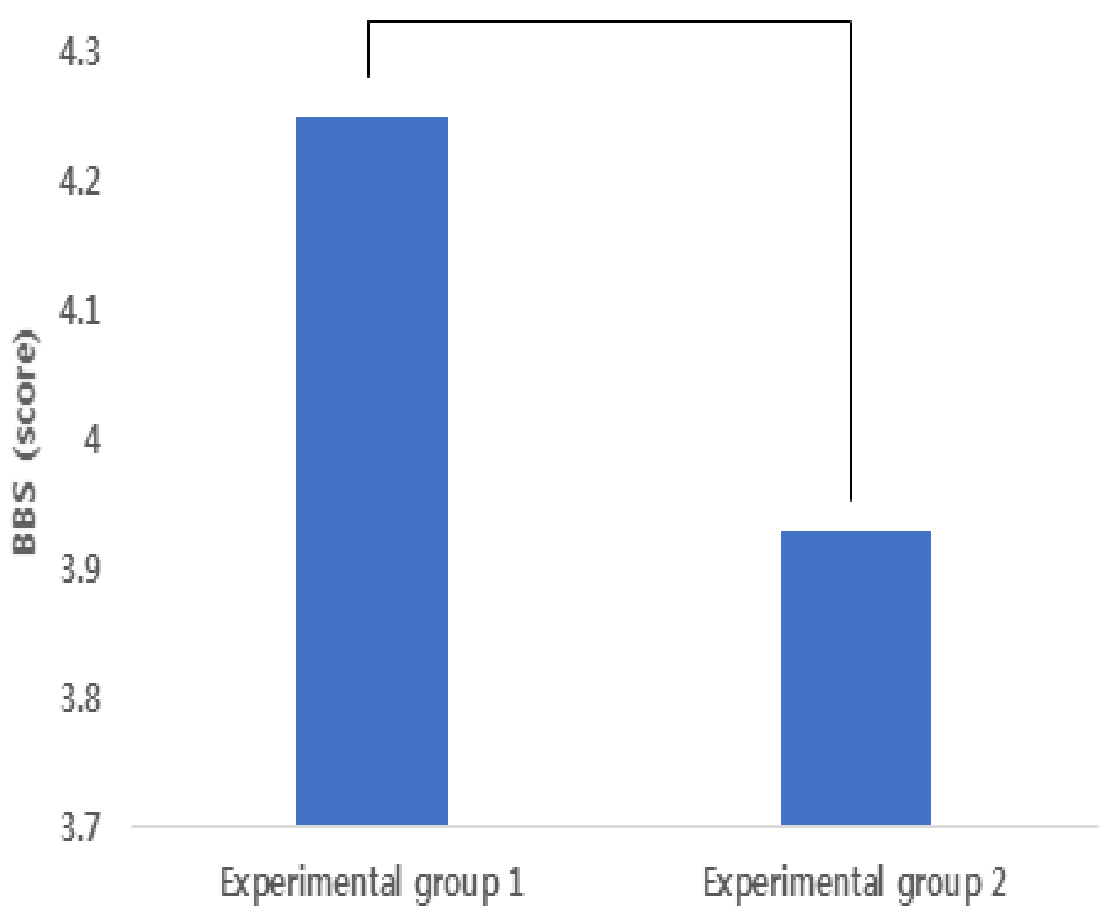

Figure 3. Balance

${ }^{*} p<0.5$, Mean $\pm S D$ : Mean \pm standard deviation, BBS: Berg balance scale

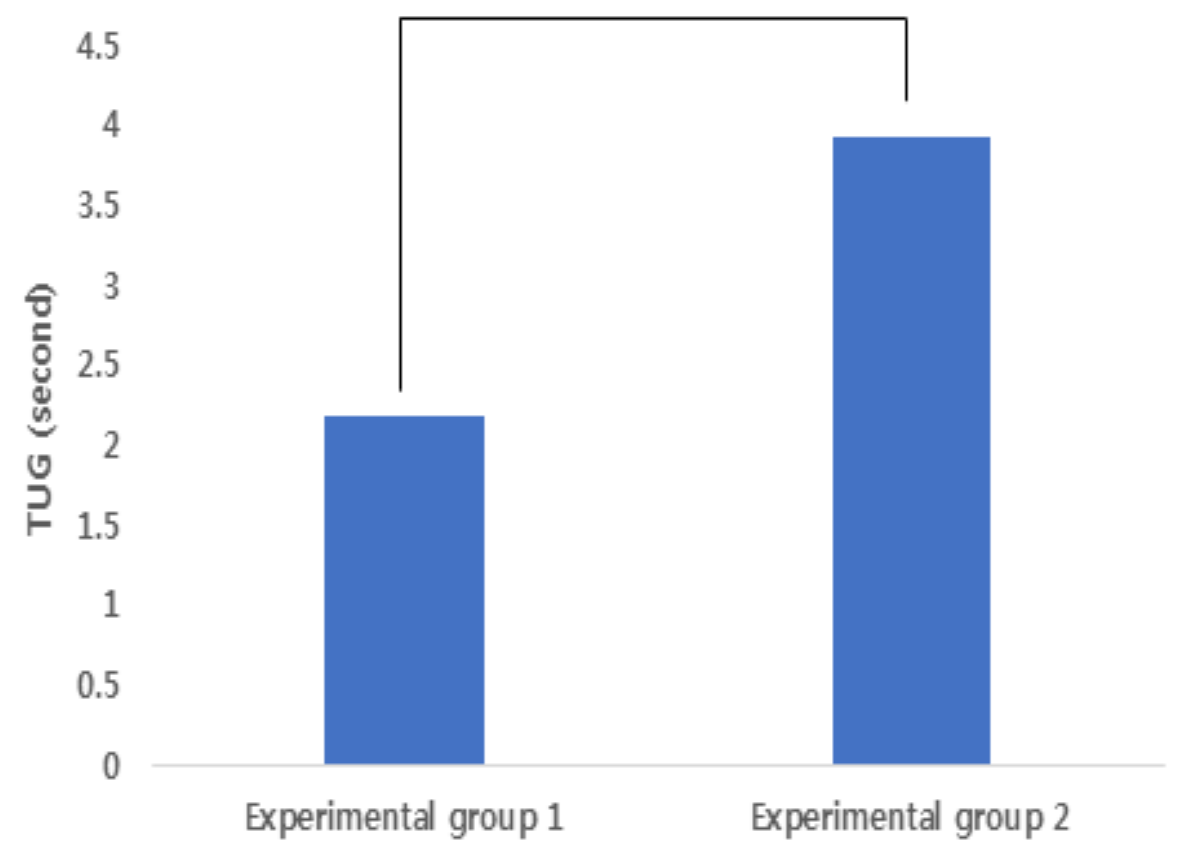

Figure 4. Gait

${ }^{*} p<0.5$, Mean $\pm S D$ : Mean \pm standard deviation, TUG: Time up and go 


\section{Discussion}

The aging-related problems are mostly associated with a decline in physical function, and most elderly people have problems with muscles and bones, experiencing diseases of the musculoskeletal system [7]. According to recent studies, muscle strength and muscle mass decrease in the elderly, and muscle strength, in particular, decrease significantly in both men and women after 65 years of age, with women reportedly experiencing a more severe decrease in muscle strength [8].

The ability to maintain balance is crucial for humans to perform purposeful activities of daily living, and it refers to the process of continuously maintaining postural stability. For an appropriate balance reaction to occur, proper mechanisms of support of the musculoskeletal system, motor functions through coordination, and integration of sensory functions are required [9].

It is difficult for elderly people to maintain body balance due to musculoskeletal dysfunction, the decline in sensory function, and postural instability, and a decrease in balance and functional mobility is a major cause of decreased gait ability and falls [10].

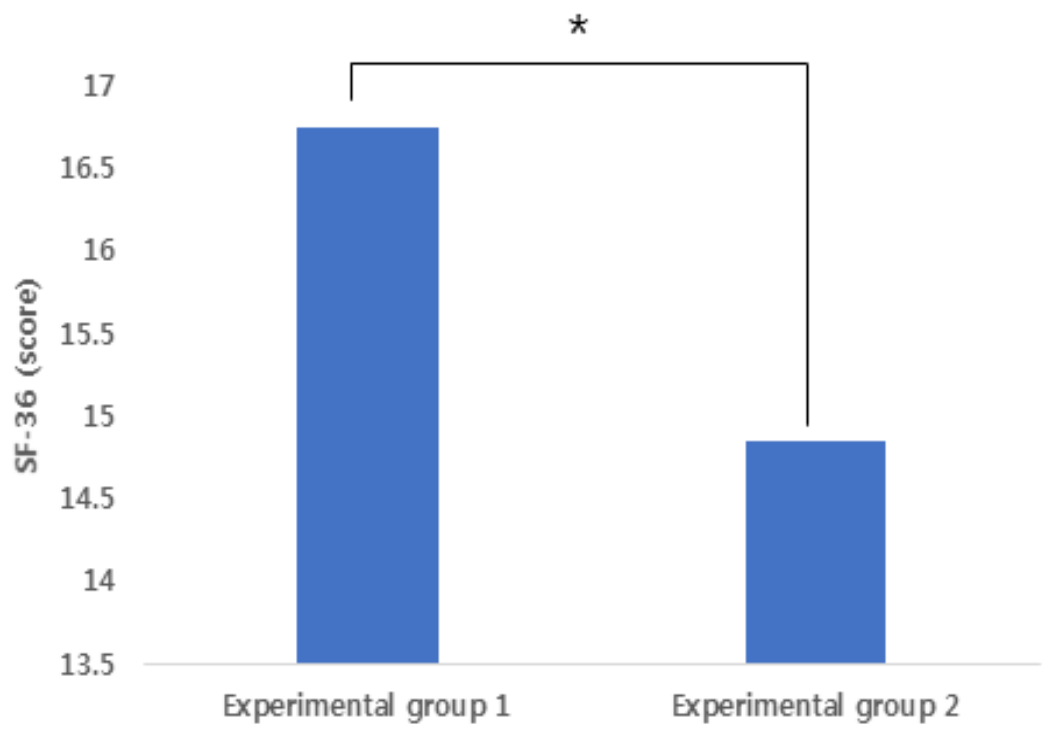

Figure 5. Quality of life

${ }^{*} p<0.5$, Mean $\pm S D:$ Mean \pm standard deviation

Among the factors that reduce balance ability in the elderly, a decrease in muscle strength and range of motion induces loss of functional independence, increasing the risk of falls. To prevent these problems, systematic and regular exercises are required [11]. It is known that more significant changes occur in the lower limb muscles than in the upper limb muscles. In particular, the quadriceps and soleus muscles have been reported to show a greater decrease in volume with aging compared to other muscles in the lower limbs [12].

Applying various types of resistance and tasks simultaneously for systematically accumulated comprehensive exercise programs positions this program with the potential to enhance the abilities of elderly people required for their physical activity, as well as manage their psychological factors. 


\section{Acknowledgments}

This work was supported by the National Research Foundation of Korea (NRF) grant funded by the Korean government (MSIT) (No-2019R1F1A1057731).

\section{References}

[1] C. J. Baranowski, "The quality of life of older adults with epilepsy: A systematic review," Seizure, vol.60, pp.190-197, Aug. (2018), DOI: 10.1016/j.seizure.2018.06.002

[2] J. Dimitrova et al., "Comparing the effects of an acute bout of physical exercise with an acute bout of interactive mental and physical exercise on electrophysiology and executive functioning in younger and older adults," Aging Clin. Exp. Res., vol.29, no.5, pp.959-967, Oct. (2017), DOI: 10.1007/s40520-016-0683-6

[3] P. G. Lee, E. A. Jackson, and C. R. Richardson, "Exercise prescriptions in older adults," Am. Fam. Physician, vol.95, no.7, pp.425-432, Apr. (2017)

[4] N. Rugbeer, S. Ramklass, A. Mckune, and J. van Heerden, "The effect of group exercise frequency on healthrelated quality of life in institutionalized elderly," Pan Afr. Med. J., vol.26, pp.35, (2017), DOI: 10.11604/pamj.2017.26.35.10518

[5] R. McDermott-Levy, A. M. Kolanowski, D. M. Fick, and M. E. Mann, “Addressing the health risks of climate change in older adults,” J. Gerontol. Nurs., vol.45, no.11, pp.21-29, Nov. (2019) DOI: 10.3928/0098913420191011-04

[6] R. W. Bohannon and A. W. Andrews, "Normal walking speed: A descriptive meta-analysis," Physiotherapy, vol.97, no.3, pp.182-189, Sept. (2011), DOI: 10.1016/j.physio.2010.12.004

[7] F. C. Vechin et al., "Comparisons between low-intensity resistance training with blood flow restriction and high-intensity resistance training on quadriceps muscle mass and strength in elderly," J. Strength Cond. Res., vol.29, no.4, pp.1071-1076, Apr. (2015), DOI: 10.1519/JSC.0000000000000703

[8] M. Á. De la Cámara, S. Higueras-Fresnillo, K. P. Sadarangani, I. Esteban-Cornejo, D. Martinez-Gomez, and Ó. L. Veiga, "Clinical and ambulatory gait speed in older adults: Associations with several physicals, mental, and cognitive health outcomes," Phys. Ther., vol.100, no.4, pp.718-727, Apr. (2020), DOI: 10.1093/ptj/pzz186

[9] F. Trombini-Souza, M. de Maio Nascimento, T. F. A. da Silva, R. C. de Araújo, M. R. Perracini, and I. C. N. Sacco, "Dual-task training with progression from variable to fixed-priority instructions versus dual-task training with variable-priority on gait speed in community-dwelling older adults: A protocol for a randomized controlled trial: Variable- and fixed-priority dual-task for older adults,” BMC Geriatr., vol.20, no.1, pp.76, Feb. (2020), DOI: 10.1186/s12877-020-1479-2

[10] Y. C. Tseng, S. H. Y. Liu, M. F. Lou, and G. S. Huang, "Quality of life in older adults with sensory impairments: A systematic review,” Qual. Life Res. Int. J. Qual. Life Asp. Treat. Care Rehabil., vol.27, no.8, pp.1957-1971, Aug. (2018), DOI: 10.1007/s11136-018-1799-2

[11] H. W. Lach, B. E. Harrison, and S. Phongphanngam, "Falls and fall prevention in older adults with early-stage dementia: An integrative review," Res. Gerontol. Nurs., vol.10, no.3, pp.139-148, May (2017), DOI: 10.3928/19404921-20160908-01

[12] E. Kovács, I. Sztruhár Jónásné, C. K. Karóczi, A. Korpos, and T. Gondos, "Effects of a multimodal exercise program on balance, functional mobility and fall risk in older adults with cognitive impairment: A randomized controlled single-blind study,” Eur. J. Phys. Rehabil. Med., vol.49, no.5, pp.639-648, Oct. (2013) 
The Impacts of a Systematic Exercise Method on Physical Activity and Psychological Factors

This page is empty by intention. 